BASU, S. N. (1952). J. gen. Microbiol. 6, 199-204.

\title{
Chaetomium brasiliensis Batista \& Pontual; Nutritional Requirements for Growth and Fruiting
}

\author{
By S. N. BASU \\ Microbiology Department, Indian Jute Mills Association Research Institute, \\ Calcutta
}

SUMMARY: Chaetomium brasiliensis requires aneurin for growth, and calcium (strontium or barium), aneurin and a low nutrient level for the formation of ascospores. Decreasing suboptimal doses of calcium progressively decrease the number of fertile perithecia until none is formed, the average perithecial size is reduced, but not the total number of perithecia. Above the optimum level, calcium delays formation of perithecia and ascospores and, ultimately, spore germination; it has no favourable effect on vegetative growth. The fungus appears able to synthesize aneurin slowly after growth is complete.

Lilly \& Barnett (1949) found that Chaetomium convolutum needs external supplies of both aneurin and biotin for growth and for ascospore formation. Apart from this, no essential vitamin requirements of Chaetomium species are known, although $C$. cochliodes fruits better in the presence of aneurin (Hawker, 1942) and that C. globosum fruits more quickly with added biotin (Buston \& Basu, 1948). C. globosum and several other Chaetomium species, however, require calcium for the formation of mature perithecia and ascospores (Basu, 1951). In this paper it is shown that the recently discovered C. brasiliensis needs calcium for fruiting, and aneurin for both growth and fruiting.

\section{MATERIALS AND METHODS}

The test organism (IJMARI 117) was undescribed when first isolated by us from rotting jute fabric. According to the Commonwealth Mycological Institute, Kew, to which a subculture was submitted, it is identical with the fungus described by Batista \& Pontual (1948) as C. brasiliensis and by Ames (1949) as C. velutinum. A subculture of the latter was kindly sent us by Dr Ames and we found it to be morphologically indistinguishable from our strain.

The organism was grown on malt agar slopes at $30^{\circ}$ and stored in the refrigerator. The basal medium used was Czapek-Dox salt solution at $\mathrm{pH} 7 \cdot 0$ and contained: $\mathrm{NaNO}_{3}, 2 \mathrm{~g}$.; $\mathrm{K}_{2} \mathrm{HPO}_{4}, 0.75$ g.; $\mathrm{KH}_{2} \mathrm{PO}_{4}, 0.25$ g.; $\mathrm{KCl}, 0.5$ g.; $\mathrm{MgSO}_{4} \cdot 7 \mathrm{H}_{2} \mathrm{O}, 0.5 \mathrm{~g}$; ; water, $1 \mathrm{l}$. The source of carbon was $\mathbf{0} \cdot \mathbf{2} \%$ glucose $(\mathrm{w} / \mathrm{v})$, unless otherwise mentioned. The medium was first prepared with half the required quantity of water and $5 \mathrm{ml}$. placed in $75 \mathrm{ml}$. American-made Pyrex conical flasks, which were cleaned before each test with hot chromicsulphuric acid. After addition of supplements, the volume of medium in each flask was made to $10 \mathrm{ml}$. with distilled water. The flasks were sterilized at $5 \mathrm{lb}$./sq.in. for $10 \mathrm{~min}$. Aneurin (as hydrochloride) was added before steriliza- 
tion as a freshly prepared solution. Calcium was added as $\mathrm{CaCl}_{2}$. Flasks were inoculated with a loopful of spore suspension and incubated at $30^{\circ}$.

As the organism produced numerous small, densely aggregated perithecia, counting perithecia was not possible. This was of little consequence because, unlike $C$. globosum and the other species previously examined, the number of perithecia, when these were formed, was always of the same order; differences were noticed in fertility and size of perithecia and in their terminal hairs. Whenever practicable cultures were duplicated.

\section{RESULTS}

\section{Effect of aneurin on growth}

On malt extract medium or on basal medium plus jute extract the fungus grew and fruited well, forming a deep smoky-black mycelium closely covered with blue-green perithecia having spirally curved terminal hairs; on basal medium alone growth did not proceed much beyond spore germination (Pl. 1, fig. 1), indicating a nutritional deficiency. Aneurin, biotin, riboflavin, pyridoxin, nicotinic acid and $\mathrm{Ca}$ pantothenate were added at concentrations of $0 \cdot 1,0.01,0.001 \mu \mathrm{g}$./flask. The fungus responded only to aneurin, growth proceeding to completion with $0.01 \mu \mathrm{g}$. but not with $0.001 \mu \mathrm{g}$. aneurin/flask. At the lower concentration some sugar remained, the mycelium was white and no perithecia were formed; at the higher concentration sugar was exhausted, the mycelium turned black and its surface was extensively covered with greyish green perithecia which, however, showed more or less straight hairs, did not form either asci or ascospores and may be described as infertile. This state of growth (see PI. 1, figs. 2 and 3), was not altered with increased doses of aneurin or combination of aneurin with any of the other substances mentioned above (at $\mathbf{0 . 2} \mu \mathrm{g}$./flask). A nutritionally adequate medium gives the same type of vegetative growth, but the perithecia, in addition to being fertile and covered with spirally curved hairs (Pl. 1, fig. 4), are darker and bigger.

The response of vegetative growth to increasing doses of aneurin was tested on basal medium which contained $3 \%(\mathrm{w} / \mathrm{v})$ glucose and $0.2 \mathrm{~m}$-phosphate buffer, and was adjusted to $\mathrm{pH} 6 \cdot 0$, the flasks being sterilized at $3 \mathrm{lb}$./sq.in. for 10 min. After growth the $\mathrm{pH}$ was still below $7 \cdot 0$. Buffer did not seem to affect growth or germination. Although glucose was still present, growth stopped in all flasks at the end of 20 days. The mycelia were washed once in distilled water, pressed lightly between filter-papers, dried at $110^{\circ}$ for $8 \mathrm{hr}$. and, after cooling in a desiccator, weighed. Maximum growth, c. $75 \mathrm{mg}$. mycelium/flask, was obtained with $0 \cdot 125 \mu \mathrm{g}$. aneurin $\mathrm{HCl} /$ flask, and half maximum growth at c. 0.04 $\mu \mathrm{g}$. aneurin $\mathrm{HCl} /$ flask (see also Table 2). The mycelium was somewhat paler at low aneurin levels, darkening being absent when the amount of growth was very small. No distinct perithecia were formed. As infertile perithecia were formed on the $0.2 \%$ glucose medium, both with and without phosphate buffer, in the latter case the final $\mathrm{pH}$ was $\mathbf{8 \cdot 2}$, it seems that the appearance of perithecia is dependent on the concentration. of nutrients remaining in the medium rather than on its $\mathrm{pH}$. 


\section{Requirements for fruiting}

Calcium. Production of ascospores was induced by adding $\mathrm{Ca}$ to the medium, the results of one experiment being summarized in Table 1. Slight precipitates were observed in flasks containing 30 p.p.m. or more of $\mathrm{Ca}$, but these disappeared as growth proceeded. At the end of the incubation period (30 days), no sugar was left in any flask. Perithecia were usually spherical, sometimes oval. Infertile perithecia were recognized from their smaller size, paler colour and deficiency in coiled hairs.

Table 1. Effect of varying concentrations of calcium on perithecial development of Chaetomium brasiliensis

(1 $\mu$ g. aneurin $\mathrm{HCl} /$ flask present throughout.)

\begin{tabular}{|c|c|c|c|c|}
\hline \multirow[b]{3}{*}{$\mathrm{Ca}$ (p.p.m.) } & \multirow{2}{*}{\multicolumn{2}{|c|}{ Day of appearance of }} & \multicolumn{2}{|c|}{ Perithecia after 30 days } \\
\hline & & & \multirow{2}{*}{$\begin{array}{c}\text { Mean length } \\
(\mu .)\end{array}$} & \multirow{2}{*}{$\begin{array}{c}\text { Infertile } \\
(\%)\end{array}$} \\
\hline & Perithecia & Ascospores & & \\
\hline $\mathbf{0}$ & 9 & np & 62 & 100 \\
\hline $0 \cdot 1$ & 9 & np & 82 & 100 \\
\hline 0.4 & 9 & 11 & 92 & 64 \\
\hline 1 & 9 & 11 & 87 & 48 \\
\hline 3 & 9 & 11 & 98 & 25 \\
\hline 5 & 9 & 11 & 100 & 20 \\
\hline
\end{tabular}

Above Ca 5 p.p.m. the days of appearance of perithecia and ascospores respectively were: at 20 p.p.m. 9 and 11 ; at 50 p.p.m. 12, 14 and 14, 16 (duplicates); at 1000 p.p.m. 13, 14 and 15, 16 (duplicates).

$\mathrm{np}=$ none produced.

$\mathrm{Ca}$ increased the size of the perithecia and the number of fertile perithecia, the optimum Ca concentration being 3-5 p.p.m.; higher doses not significantly affecting these characteristics. No differences were detected in the degree of fertility, i.e. the number of spores per fertile perithecium. Above the optimum Ca range, other effects were noticed, the most important of which was delay in the maturation of perithecia. At Ca 30 p.p.m. and greater, the fungus began growth as white flakes and not as the normal mat but this difference soon vanished. At Ca 1000 p.p.m., germination was delayed by 2 days and the perithecia appeared as a scattered overgrowth and were probably fewer in number. At the concentrations tried, $\mathrm{Ca}$ did not affect the rate of growth or the total amount of growth (mycelium dry-weight) and had no effect without aneurin in the medium. With the formation of spores the colour of the perithecia changed to a deeper shade, from grey-green to blue-green, this colour change being an accurate indication of perithecial fertility.

In previous experiments with C. globosum, Ca was found to be replaceable by $\mathrm{Sr}$ and $\mathrm{Ba}$, although particularly with $\mathrm{Ba}$ the number of mature perithecia formed was fewer (Basu, 1951). These elements also induced fruiting in C. brasiliensis, the effects being equal to that of $\mathrm{Ca}$, when $\mathrm{Sr}$ and $\mathrm{Ba}$ were supplied at 10 p.p.m. (as nitrate and chloride respectively).

When Ca 10 p.p.m. was added, after completion of growth, to flasks initially without $\mathrm{Ca}$ and containing $\mathrm{l} \mu \mathrm{g}$. aneurin, there followed quickly a deepening 
of colour and formation of spores, the final result being the same as when $\mathrm{Ca}$ was added before inoculation. Thus Ca was concerned only with the process of fruiting and not with growth. The strain from Ames was also tested; the responses were the same as with the usual test organism.

Addition of a hot-water extract of jute to the basal medium caused good growth and fruiting. $\mathrm{Mn}, \mathrm{Fe}, \mathrm{Co}, \mathrm{Ni}, \mathrm{Sn}$, and $\mathrm{Pb}$, found in the ash of jute extract by Basu (1951), were tested at 1 and 10 p.p.m. in the medium but no effect on growth or fruiting was observed; Co and Ni were inhibitory to growth.

Table 2. Effect of aneurin on fruiting of Chaetomium brasiliensis

(Ca 10 p.p.m. present throughout.)

\begin{tabular}{|c|}
\hline $\begin{array}{c}\text { Aneurin } \mathbf{H C l} \\
(\mu \mathrm{g} . / \text { flask })\end{array}$ \\
\hline 0.001 \\
\hline 0.003 \\
\hline 0.006 \\
\hline 0.010 \\
\hline $0 \cdot 020$ \\
\hline 0.030 \\
\hline 0.040 \\
\hline 0.050 \\
\hline 0.075 \\
\hline $0 \cdot 100$ \\
\hline
\end{tabular}

$\begin{array}{cc}\overbrace{\text { Perithecia }}^{\text {Day of appearance of }} \\ \text { np } & \text { Ascospores } \\ n p & n p \\ n p & n p \\ 26 & \text { np } \\ 20 & 30 \\ 18 & 23 \\ 11 & 21 \\ 9 & 17 \\ 9 & 11 \\ 9 & 11 \\ & 11\end{array}$

Glucose remaining after 30 days' incubation were $9 \cdot 1,6 \cdot 1$ and $<2 \cdot 0 \mathrm{mg}$./flask with $0 \cdot 001$, 0.003 and $0.006 \mu \mathrm{g}$. (or higher) aneurin $\mathrm{HCl} /$ flask.

$\mathrm{np}=$ none produced.

When Whatman no. 5 filter-paper was used as the source of carbon instead of glucose and aneurin was supplied, C. brasiliensis, like C. globosum, formed many fertile perithecia in 7 days without added Ca. Fewer fertile perithecia formed by $C$. brasiliensis with Whatman no. 30 and no. 44 filter-papers, which have progressively smaller ash contents, only a few spores being formed with no. 44, although they appeared equally quickly with each filter-paper tried. That $C$. globosum fruited equally well on all these filter-papers is probably related to the fact that, unlike $C$. brasiliensis, it sporulates to some extent on a medium with a very low initial glucose content. The reaction of $C$. brasiliensis to the different filter-papers was reproduced when equivalent quantities of their ash were added to the usual glucose medium. Ordinary macro-chemical analysis showed $\mathrm{Ca}$, but not $\mathrm{Sr}$ or $\mathrm{Ba}$, in the ash of no. 5 filter-paper. Cellulose decomposition of filter-paper, determined by weighing the residue of no. 5 filter-paper left after 13 days' growth, was the same with both species, with or without $\mathrm{Ca} 10$ p.p.m. added to the medium, which is consistent with the observation that $\mathrm{Ca}$ does not affect vegetative growth.

Aneurin. In Table 2 are given the results of an experiment designed to see whether aneurin was necessary for fruiting, apart from growth. Germination and rate of growth of mycelium did not appear to vary with the dosage of aneurin. Fruiting was progressively delayed below $0.05 \mu \mathrm{g}$./flask and at $0.006 \mu \mathrm{g}$./flask no perithecia were formed in 30 days, although growth had 
reached completion. It seems that a much higher concentration of aneurin is needed for fruiting than for growth and that the fungus can slowly synthesize the extra amount after growth is complete, when a little more aneurin than needed to reach this stage is originally present. The average size of perithecia and the percentage of infertile perithecia at 45 days did not differ significantly between flasks and were of the same order as obtained under optimum conditions (Table 1). The same applies to the total number of perithecia, except that at $0.01 \mu \mathrm{g}$. aneurin/flask the number was smaller.

Sugar concentration. Apart from adequate amounts of $\mathrm{Ca}$ and aneurin, another requirement for fruiting is that the concentration of free sugar in the medium must be very low; this was indicated by the quicker fruiting of the fungus on filter-paper. With increasing amounts of glucose in the medium fruiting was correspondingly delayed, at the following initial concentrations of glucose $(\%)$ the days of appearance of perithecia and ascospores respectively were: $0.2 \%, 8,11 ; 0.3 \%, 10,14 ; 0.3 \%, 13-15,15-18 ; 0.5 \%, 15-18,20-21$; $\mathbf{0 . 6} \%, 21,23$. In every case less than $2 \mathrm{mg}$. glucose were left when ascospores were first seen. In another experiment, with $2 \%$ glucose, $1 \mu \mathrm{g}$. aneurin, and 20, 50 or 100 p.p.m. Ca in the medium fruiting did not take place even when only $18 \mathrm{mg}$. sugar were left after growth. Even immature perithecia are not formed, either with or without $\mathrm{Ca}$ in the medium, unless the sugar content approaches exhaustion.

\section{DISCUSSION}

A low nutrient level favours fruiting in several other species of Chaetomium (Basu \& Bose, 1950), C. convolutum (Lilly \& Barnett, 1949) and in some other Ascomycetes (Asthana \& Hawker, 1936; Hawker \& Chaudhuri, 1946). In general, environmental conditions which restrict growth favour reproduction, particularly the formation of sexual spores.

In its aneurin requirement for growth, $C$. brasiliensis resembles $C$. convolutum which is the only other Chaetomium species shown to have a specific vitamin requirement. Lilly \& Barnett (1949), examining the effect of aneurin on $C$. convolutum, found that at a given nutrient concentration, increasing doses of aneurin successively initiated vegetative growth, formation of perithecia and of ascospores; within certain limits, the number of mature perithecia and ascospores increased. With $C$. brasiliensis more aneurin is needed for the formation of perithecia than for complete vegetative growth, and when enough calcium is present fruiting always follows the appearance of perithecia. The only other noticeable effect of aneurin on this species was in the acceleration of the appearance of perithecia and ascospores. C.brasiliensis does not require an external supply of biotin, which has the same effect as aneurin on $C$. convolutum.

Instances of the stimulation of spore formation by calcium are rare. Brian \& Hemming (1950) found that conidia formation by the imperfect fungus Trichoderma viride was stimulated by 0.01 to $0.1 \%$ calcium chloride. Stimulation of sexual spore formation by calcium, strontium or barium is known only in other species of Chaetomium, in particular C. globosum (Basu, 1951). The 
replaceability of calcium by strontium or barium is even more marked in C. brasiliensis than in $C$. globosum, but manganese, which engendered a few fertile perithecia in the latter, had no effect on the former. Similarity with C. globosum is also observed in that calcium has no effect on vegetative growth and both species react to calcium even when added after complete growth. However, from a consideration of the two strains of $C$. brasiliensis and the three strains of $C$. globosum tested, a rather fundamental point of difference is noted between the two species, that while in $C$. brasiliensis calcium induces only the formation of ascospores in the large number of infertile perithecia which develop even without the metal, in C. globosum the transition is from a few or no perithecia, infertile or fertile, to a large number of fertile perithecia.

I wish to thank the Research Director, Indian Jute Mills Association Research Institute, for permission to publish these results.

\section{REFERENCES}

Ames, L. M. (1949). New cellulose destroying fungi isolated from military material and equipment. Mycologia, 41, 637.

Asthana, R. P. \& Hawker, L. E. (1936). The influence of certain fungi on the sporulation of Melanospora destruens Shear and of some other Ascomycetes. Ann. Bot., Lond. 50, 325.

BASU, S. N. (1951). Significance of calcium in the fruiting of Chatomium species, particularly Chaetomium globosum. J. gen. Microbiol. 5, 231.

Basu, S. N. \& Bose, R. G. (1950). Factors affecting the fruiting of Chaetomium species. J. gen. Microbiol. 4, 132.

Batista, A. C. \& Pontual, D. (1948). Alguns fungos do gênero Chaetomium. Bol. Sec. Agr. Indús. Com. Pernambuco, B, 15, 62.

Brian, P. W. \& Hemming, H. G. (1950). Some nutritional conditions affecting spore production by Trichoderma viride Pers. ex Fries. Trans. Brit. mycol. Soc. 33, 132.

Buston, H. W. \& BASU, S. N. (1948). Some factors affecting the growth and sporulation of Chaetomium globosum and Memnoniella echinata.J. gen. Microbiol. $2,162$.

HAwKER, L. E. (1942). Effect of vitamin $B_{1}$ on concentration of glucose optimal for certain fungi. Ann. Bot., Lond. N.S. 6, 631.

Hawker, L. E. \& Chaudhuri, S. D. (1946). Growth and fruiting of certain ascomycetous fungi as influenced by the nature and concentration of carbohydrate in the medium. Ann. Bot., Lond. N.S. 10, 185.

LILly, V. G. \& BARNETT, H. L. (1949). The influence of concentration of nutrients, thiamin, and biotin upon growth, and formation of perithecia and ascospores of Chaetomium convolutum. Mycologia, 41, 186.

\section{EXPLANATION OF PLATE}

Fig. 1. Culture of C. brasiliensis after 14 days on the basal medium. $\times 1 \cdot 4$.

Fig. 2. Culture of $C$. brasiliensis after 14 days on the basal medium plus $1 \mu \mathrm{g}$. aneurin, showing densely aggregated infertile perithecia. $\times 1 \cdot 4$.

Fig. 3. Crushed perithecium of $C$. brasiliensis after 20 days on basal medium plus $1 \mu \mathrm{g}$. aneurin, showing absence of asci, ascospores or curved terminal hairs. $\times \mathbf{2 5 0}$.

Fig. 4. Crushed perithecium of $C$. brasiliensis after 14, days on basal medium plus $1 \mu \mathrm{g}$. aneurin and 5 p.p.m. calcium, showing cylindrical asci, ascospores, and curved terminal hairs. $\times \mathbf{2 5 0}$. 
Journal of General Microbiology, Vol. 6, Nos. 3 and 4
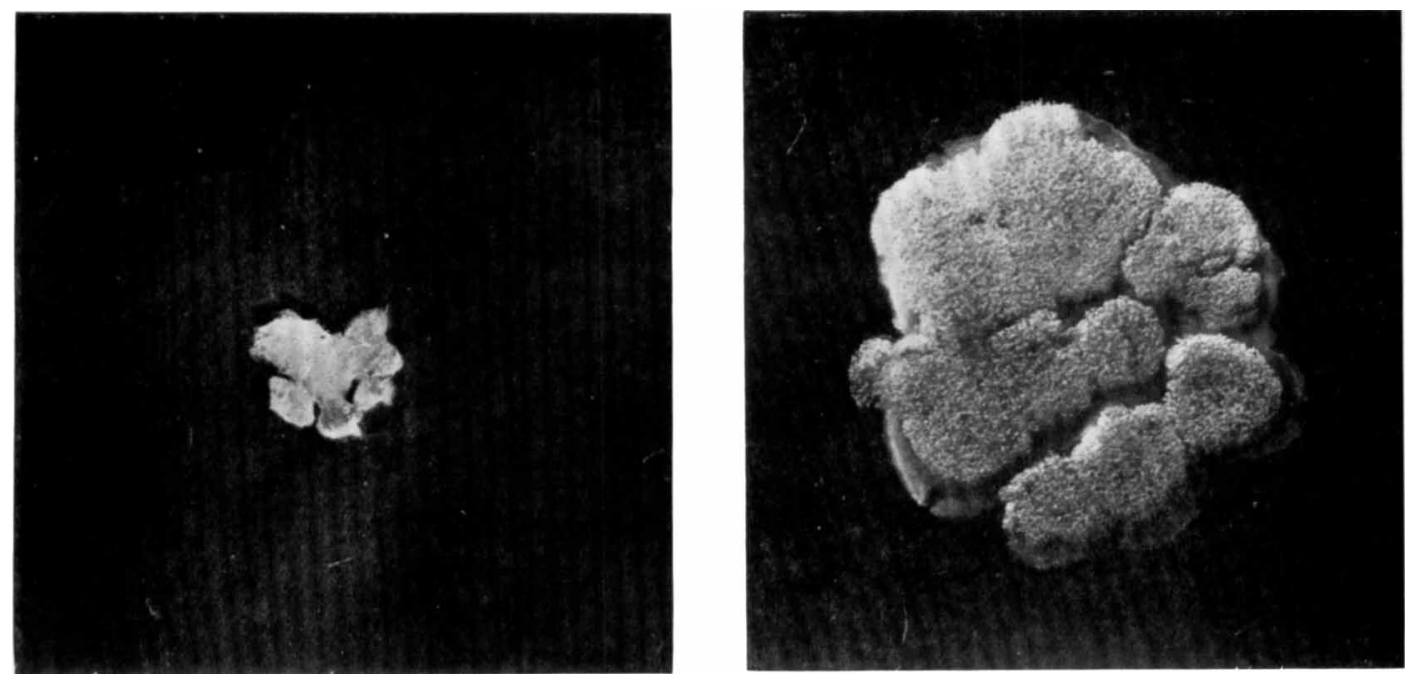

Fig. 1

Fig. 2

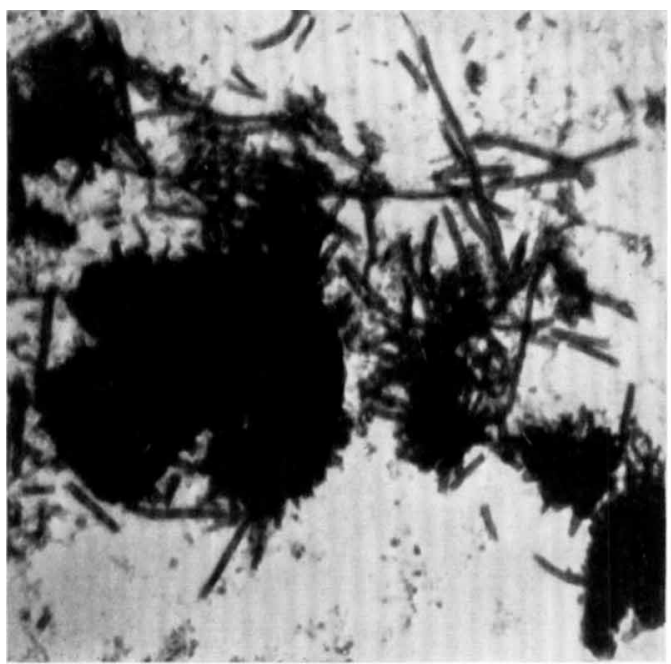

Fig. 3



Fig. 4

S. N. Basu-Nutrition of Chaetomium brasiliegsis. Plate 1 